\title{
Commissioning of the ATLAS Tile calorimeter with Single Beam and First collisions
}

\author{
J. G. Saraiva a b * \\ On behalf of the ATLAS Tile Calorimeter group \\ ${ }^{a}$ LIP, Av Elias Garcia 14 1, 1000-149 Lisboa, Portugal (Main Address)

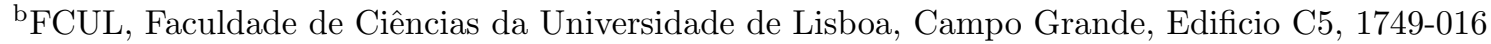 \\ Lisboa, Portugal
}

The ATLAS hadronic barrel calorimeter was tested using dedicated calibration systems, cosmic ray muons and single beam data prior to the start of collisions. Before collisions, in addition to the hardware maintenance, the verification of the electromagnetic energy scale set during test-beam and the read-out synchronization of the tile calorimeter were undertaken. The results obtained showed that the calorimeter was well calibrated and ready for the challenges of the LHC collisions.

\section{Introduction}

The Tile Calorimeter [1] (TileCal) is the barrel hadronic calorimeter of the ATLAS detector [2]. Within the ATLAS physics program, the TileCal plays an fundamental role in jets and missing $E_{T}$ measurements. The biggest challenges will be to guarantee a $1 \%$ accuracy in the jet energy scale for the top quark mass measurement and the searches for new physics where an accurate account of the missing $E_{T}$ is of great importance. The energy loss by muons in TileCal is also used as an input to muon reconstruction and for background suppression in processes like $H \rightarrow Z Z^{*} \rightarrow 4 l(4 \mu, 2 e 2 \mu)$ [3]. During the past few years TileCal has been probed using its dedicated calibration tools (see [4] also in this proceedings): charge injection, laser and cesium systems. Performance studies were also performed with data acquired using cosmic rays and single beam. The major achievements from this precollisions era with high energy particles as well as results from the first collisions are summarized in this paper.

\footnotetext{
*Work financed in part by FCT (Portugal), co-financed by QREN/POHP and QREN/COMPETE of European Union ESF and ERDF funds
}

\section{The ATLAS Tile calorimeter}

TileCal is a sampling calorimeter with scintillating plastic tiles embedded in a steel matrix. The scintillating tiles are grouped in cells read out by a pair of photomultipliers (PMTs). Wavelength shifter optical fibers carry the light from cells to PMTs.

Three cylinders, each made from 64 modules, cover the region $|\eta|<1.7$. Within this volume there are four partitions, with independent electronic and readout chains, $\mathrm{EBC}$ and $\mathrm{EBA}$ in the outer cylinders and LBC and LBA in the central cylinder. Each module has three radial layers: A, $\mathrm{BC}$ and $\mathrm{D}$. The cells distribution follows a projective geometry resulting in a segmentation for the A and BC radial layer of $\Delta \eta \times \Delta \phi=0.1 \times 0.1$ and for the $\mathrm{D}$ radial layer of $\Delta \eta \times \Delta \phi=0.2 \times 0.1$.

There are a total of 5182 instrumented cells in TileCal. Currently (7-May-2010) $97.1 \%$ of the cells are operational in physics runs. Of the cells not used in physics $2.6 \%$ are off due to low voltage power supplies problems. The physics signal is reconstructed using the optimal filter algorithm [5] that requires a good synchronization of the TileCal cells to have an online reconstruction with $1 \%$ energy precision [4]. A detailed study of the noise description has shown that TileCal noise is 
characterized by non-gaussian tails and a double gaussian description is needed in order to avoid an excess of the number of reconstructed jet clusters $[6]$.

\section{Energy scale from cosmic ray muons}

The TileCal energy scale was verified using cosmic ray muons [6]. The muons selected used tracks reconstructed in the inner detector. The tracks are extrapolated to the TileCal radial layers to calculate the crossed path length per cell. The energy loss per unit path length crossed by the muon track, $d E / d x(\mathrm{MeV} / \mathrm{mm})$, is calculated. The results obtained for each radial layer are shown in Figure 1 including also the corresponding measurement performed on test beam data [7]. The energy scale shows an agreement between radial layers within the measurements uncertainty of about $4 \%$. The measured energy scale has an agreement with earlier testbeam results with ratios of $1.01 \pm 0.03$ for A layer, $0.96 \pm 0.04$ for $\mathrm{BC}$ layer and $0.98 \pm 0.03$ for the $\mathrm{D}$ layer. The uniformity in $\eta$ and $\phi$ has also been studied and shows a maximum deviation of $3 \%$ from the average.

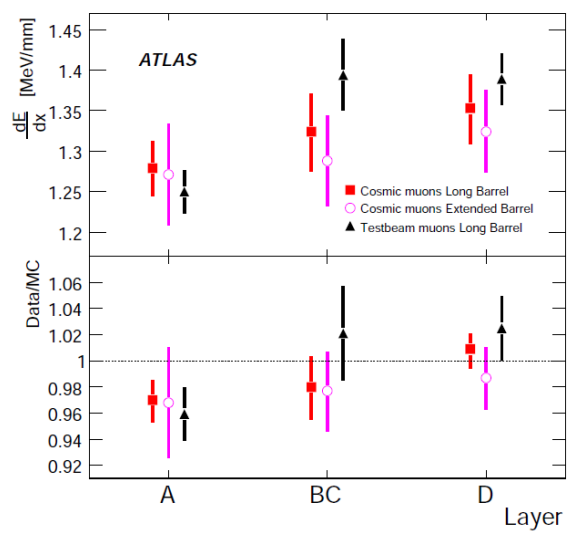

Figure 1. $\mathrm{dE} / \mathrm{dx}$ for cosmic and testbeam muons shown per radial layer compared to Monte Carlo. Error bars combine in quadrature both statistical and systematic uncertainties.

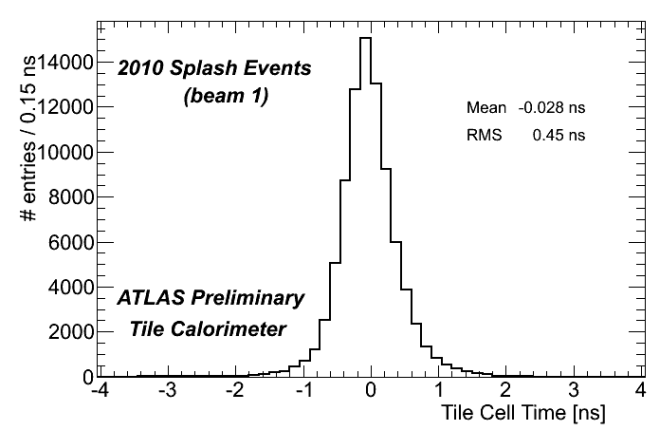

Figure 2. TileCal cells time. Splash events result from dumping the proton beam on the tertiary collimator in front ATLAS. The splash events lead to very large energy deposits that corresponds to the regime where TileCal timing resolution is the highest.

\section{Read-out synchronization}

The read-out synchronization of the TileCal was first made using the laser system. At that stage the synchronization was about $2 \mathrm{~ns}$ in each TileCal partition. The result was verified with cosmic ray muons [8] and single beam data in 2008 [9]. The two measurements agreed within about 2 ns [8]. These studies with both cosmic ray muons and single beam data (splash events) allowed to measure the remaining global delays of about 10 ns between the four TileCal partitions. The detector was synchronized using the single beam 2008 data achieving a precision better than 2 ns. Later in 2009 with the beam startup, another series of single beam runs were made and the read-out synchronization status was updated. In 2010 using the 2009 Single beam data the calorimeter was successfully synchronized to a precision better than $1 \mathrm{~ns}$ (Figure 2).

\section{First Collisions}

In November 2009 the Large Hadron Collision entered a commissioning phase to reach the optimal operating conditions. From November 2009 up to now the center of mass energy of the proton- 


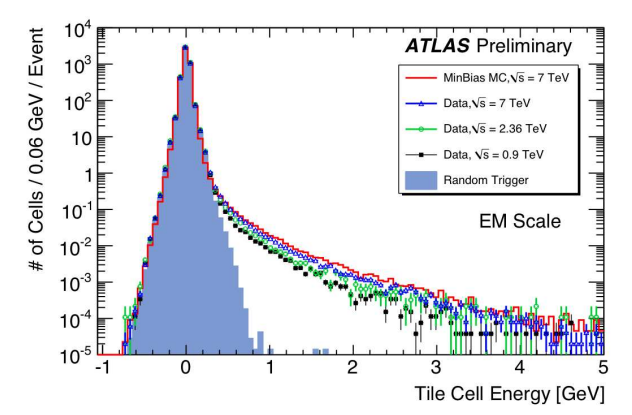

Figure 3. Cell energy distribution for collisions at different $\sqrt{s}$, for randomly triggered events (filled histogram) and for Monte-Carlo at $\sqrt{s}=7 \mathrm{TeV}$.

proton collisions was increased from $900 \mathrm{GeV}$, corresponding to protons colliding at the injection energy, to $2.36 \mathrm{TeV}$ and finally up to $7 \mathrm{TeV}$. In Figure 3 the energy using the electromagnetic scale in the TileCal cells is plotted for three values of $\sqrt{s}$. The Monte-Carlo for $\sqrt{s}=7 \mathrm{TeV}$ and the distribution resulting from random triggers are also shown. A reasonable agreement is obtained. The response in $\phi$ has an excellent agreement with Monte-Carlo (not shown). The response in $\eta$ is presenter in Figure 4 and shows that while the same general pattern is seen in both data and Monte-Carlo, there is a disagreement for $|\eta|>1.0$. The preliminary results are promising although there are still some issues to be understood.

\section{Conclusions}

TileCal performance was characterized using different types of data. The high fraction of operational photomultipliers, $97.1 \%$ as of 7-May-2010 resulted from a long and successful commissioning phase. Results from cosmic muons verified the energy scale with a precision better than $4 \%$. Combined results of the laser system, the single beam data and cosmic ray muons have contributed in 2008 to the TileCal synchronization at the level of 2 ns. In 2010, using single beam data from 2009, a 1 ns synchronization was obtained. The first re-

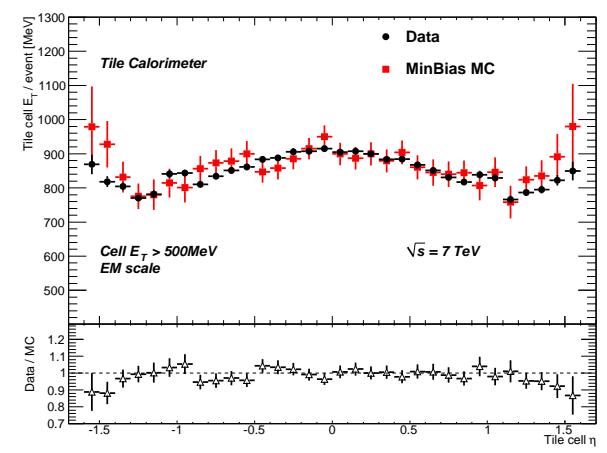

Figure 4. Average cell energy response in $\eta$ for $\sqrt{s}=7 \mathrm{TeV}$ collisions data.

sults from collisions have shown that the detector response has a reasonable agreement with MonteCarlo. At present the LHC is producing collision at a center of mass energy equal to $7 \mathrm{TeV}$. TileCal is well calibrated, operating smoothly and ready for new challenges from collisions data.

\section{REFERENCES}

1. ATLAS/Tile Calorimeter Collaboration, Technical Design Report CERN/LHCC 96-42

2. The ATLAS Collaboration, G. Aad et al., JINST 3 (2008) S08003

3. ATLAS Collaboration, CERN-OPEN-2008020

4. R. Febbraro, in this proceedings

5. W.E.Cleland and E.G.Stern, NIMA-338, 467497, January 1994

6. The ATLAS Collaboration, The Tile Calorimeter Readiness for collisions, Submitted for publication to EPJC, 2010.

7. TileCal Collaboration, NIM A 606 (2009) 362-394

8. Summarized in [6]. Details in private communication ATL-TILECAL-INT-2010-002 CERN Geneva 2010

9. M Volpi, L Fiorini, I Korolkov, ATLTILECAL-PUB-2009-008 CERN， Geneva 2009. 